\title{
BIBLOGTECARIOS:
}

\section{EL PODER DE LAS CONEXIONES Y DEL CROWDSOURCING}

\author{
Belén Benito-Blázquez, Ana Doñate-Cifuentes, David Gómez-Domínguez, \\ Julián Marquina-Arenas y Evelio Martínez-Cañadas
}

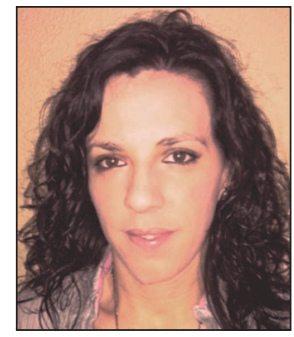

Belén Benito-Blázquez es diplomada en biblioteconomía y documentación y licenciada en documentación por la Universidad de Salamanca. En la actualidad está cursando el máster en Bibliotecas y Sistemas de Información Digital en la Universidad Carlos III de Madrid. Ha trabajado como documentalista especializada en bibliotecas y unidades de información en ciencias de la salud desde el año 2008. Es coordinadora de las redes sociales del portal especializado en biblioteconomía y documentación BiblogTecarios así como colaboradora en los diferentes artículos de la web. Está vinculada profesionalmente al Centro Nacional de Investigaciones Cardiovasculares Carlos III (CNIC) como técnico de biblioteca y documentación.

mbelenbenito@gmail.com

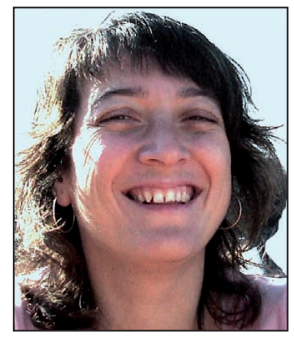

Ana Doñate-Cifuentes es licenciada en filología hispánica por la Universidad de Valencia y licenciada en documentación por la Universidad Politécnica de Valencia. En la actualidad cursa el master en bibliotecas y sistemas de información digital en la Universidad Carlos III de Madrid. Desde el año 2000 trabaja en la biblioteca de la Universidad CEU Cardenal Herrera de Moncada (Valencia) donde es la responsable de gestión de los recursos electrónicos y de la mediateca.

andoci@gmail.com

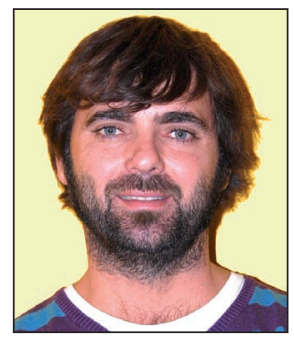

David Gómez-Domínguez es diplomado en biblioteconomía y documentación, licenciado en documentación, y diploma de estudios avanzados por la Universidad de Granada (UGR). Ha ejercido como docente en el Depto. de Biblioteconomía y Documentación el la UGR y en la Escuela Superior de Comunicación de Granada (ESCO), y ha impartido cursos de formación especializada en las principales asociaciones profesionales españolas. Trabaja como gestor del conocimiento en el Centro de Documentación del Observatorio de la Infancia en Andalucía (en lib-web.cats). Junto a Evelio Martínez-Cañadas coordina las publicaciones de la plataforma BiblogTecarios. Es voluntario digital en el proyecto Andalucía Compromiso Digital de la Consejería de Economía, Innovación y Ciencia.

davidgadir@gmail.com

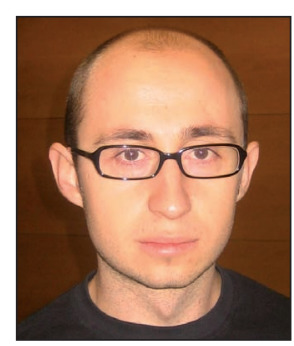

Julián Marquina-Arenas es Community Manager de Baratz-Servicios de Teledocumentación, donde se encarga de la gestión, comunicación y dinamización de las redes sociales de la empresa así como de buscar nuevas líneas de comunicación y participación. Es director de RecBib - Recursos Bibliotecarios y de la plataforma de blogs BiblogTecarios. Diplomado en biblioteconomía y documentación y licenciado en documentación por la Universidad Carlos III de Madrid. Es miembro del Consejo Editorial del Anuario ThinkEPI.

http://www.recbib.es http://www.biblogtecarios.es recbib@gmail.com

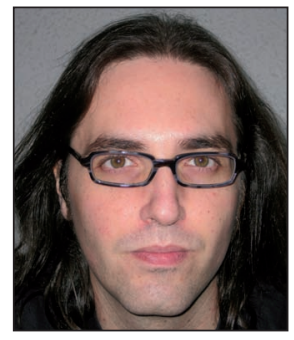

Evelio Martínez-Cañadas es licenciado en biología y diplomado en biblioteconomía y documentación por la Universitat de Barcelona. Ha ejercido como bibliotecario, documentalista y archivero en diferentes instituciones públicas y privadas. Es coordinador del equipo de gestión del archivo de infancia de la DG d'Atenció a la Infància i l'Adolescència (Dgaia) de la Generalitat de Catalunya, a través de la consultora de nuevas tecnologías Serikat.es. Es coordinador de publicaciones, junto a David Gómez-Domínguez, de la plataforma BiblogTecarios. Se especializa en recuperación y organización de información, análisis de contenido y psicología de la información. Escribe regularmente sobre ciencia cognitiva y psicología y sociología de la información.

http://www.serikat.es http://girocognitivo.wordpress.com http://eveliomartinez.wordpress.com emartibd@gmail.com

Artículo recibido el 19-08-11 Aceptación definitiva: 31-10-11 


\title{
Resumen
}

BiblogTecarios surgió con un objetivo definido: ofrecer a sus miembros la posibilidad de contar con una plataforma para publicar sus conocimientos, inquietudes u opiniones acerca de su sector profesional, el de la información y la documentación. Desde sus orígenes se vio claro que la finalidad del proyecto no iba encaminada a que unos pocos publicasen todos los días, sino que a mayor cantidad de personas participando en el proyecto mayor variedad de temas se iban a tratar. Son ya 40 los blogs dentro de BiblogTecarios, lo que hace que las herramientas de comunicación entre los miembros sean esenciales para el buen funcionamiento de la web, así como las ganas de trabajar por un proyecto conjunto (colaborativo y cooperativo). El crowdsourcing (o la inteligencia colectiva de sus miembros) ha hecho que el proyecto crezca: las conexiones personales y el trabajo colectivo son el pilar clave para seguir creciendo. BiblogTecarios es conexión entre personas, es crowdsourcing.

\section{Palabras clave}

Blogs, Crowdsourcing, Colaboración, Cooperación, Redes sociales.

\section{Title: BiblogTecarios: the power of connections and crowdsourcing}

\section{Abstract}

BiblogTecarios ("blogging librarians") was created with a clear objective: to offer its members the possibility of having a platform to publish their knowledge, concerns and opinions about their professional sector, that of Information and Documentation. Since its beginnings it has been clear that the purpose of the project was not intended to allow a few to publish each day, but rather that the more people participating in the project, the greater variety of topics could be discussed. Now that BiblogTecarios has 40 blogs, communication tools are essential for the proper functioning of the website, as well as the desire to work on a joint (collaborative and cooperative) project. Crowdsourcing, or the collective intelligence of its members, helped this project to grow; personal connections and collective work are the key pillars for its continued growth. BiblogTecarios connects people: it is crowdsourcing.
\end{abstract}

\section{Keywords}

Blogs, Crowdsourcing, Collaboration, Cooperation, Social networks.

Benito-Blázquez, Belén; Doñate-Cifuentes, Ana; Gómez-Domínguez, David; Marquina-Arenas, Julián; Martínez-Cañadas, Evelio. "BiblogTecarios: el poder de las conexiones y del crowdsourcing". El profesional de la información, 2011, noviembre-diciembre, v. 20, n. 6, pp. 677-681.

http://dx.doi.org/10.3145/epi.2011.nov.12

\section{BiblogTecarios}

\section{¿Qué es BiblogTecarios?}

Espacio web colaborativo con formato blog que nace en octubre de 2010 por iniciativa de Julián Marquina-Arenas (director de RecBib y community manager en Baratz). La plataforma ha sido creada utilizando el gestor de contenidos Drupal. Incluso con anterioridad al lanzamiento oficial de la plataforma (unos dos meses antes), el proceso de configuración del Drupal de BiblogTecarios ya reflejaba la filosofía que preside el proyecto: entre los biblogtecarios del momento (entonces un total de 22 personas) se decidió, de manera democrática, cuál sería la plantilla que sería utilizada, los colores, las categorías que iban a conformar la taxonomía, la imagen corporativa, etc.

En la actualidad el número de componentes ha crecido hasta un total de 43 estudiantes de biblioteconomía y documentación y profesionales del sector, tanto de España como de América Latina.

El objetivo último que se persigue es realizar aportaciones de calidad en lo que a nuestra área de conocimiento se refiere: para ello, cada día un biblogtecario publica un post sobre algún tema concreto del ámbito de la información y la documentación. Se trata de compartir conocimiento de manera generosa, con una filosofía 2.0 y con la intención de generar debate a partir del cual se puedan crear nuevas ideas o conocimientos.

En el proyecto participan 43 biblogtecarios, estudiantes de biblioteconomía y documentación y profesionales del sector, tanto de España como de América Latina

En este espacio web, conviven tres perfiles de usuarios:

- Usuarios individuales de BiblogTecarios, que comparten sus inquietudes y reflexiones mediante blogs personales en una página web común. En este caso, cada biblogtecario es responsable de sus propios contenidos, asumiendo un compromiso previo de regularidad en lo que a publicación se refiere.

http://www.biblogtecarios.es

- Usuario colectivo BiblogTecarios con el que se trabaja de forma colaborativa siguiendo los principios del crowd- 

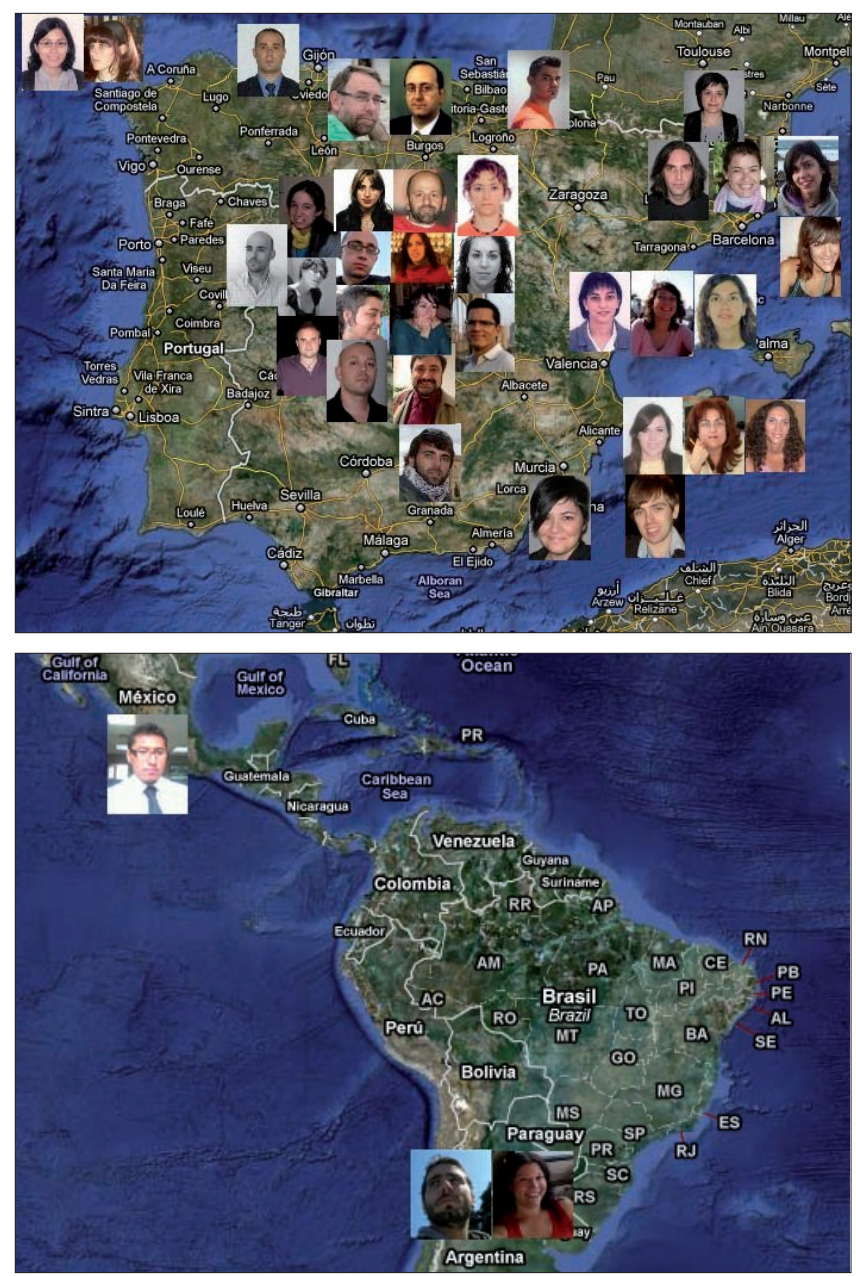

Figura 1. Mapa BiblogTecario

sourcing. El resultado final es un post consensuado entre todos los que participen de manera voluntaria en la elaboración de esa entrada colectiva, con la ayuda de las herramientas que se describirán en el apartado 2.

http://www.biblogtecarios.es/blog/biblogtecarios

- Usuario "Firmas invitadas", que ofrece la posibilidad de publicar contenidos a los profesionales/estudiantes que tienen algo puntual que contar o compartir, pero que no disponen del tiempo suficiente como para poder comprometerse a publicar en la plataforma con regularidad. http://www.biblogtecarios.es/firmas-invitadas

Se trata de compartir conocimiento de manera generosa, con una filosofía 2.0 y con la intención de generar debate

Según la RAE, cooperar consiste en obrar juntamente con otro $u$ otros para un mismo fin. Este es el espíritu de BiblogTecarios: un grupo de personas que pertenecen a una misma disciplina, pero que a su vez, se centran en una de las diversas ramas de ésta. Así pues, se pretende favorecer el enriquecimiento tanto personal como profesional de los propios miembros, y de sus lectores.

\section{Objetivos de BiblogTecarios}

La plataforma se ha fijado los siguientes objetivos:
- convertirse en un espacio virtual en el que se debaten, explican y difunden conocimientos, situaciones, tendencias..., al ritmo en que la disciplina cambia y evoluciona;

- dinamizar el entorno profesional, aportando más visibilidad tanto a profesionales como a bibliotecas, archivos y centros de documentación;

- establecer un puente entre los profesionales de España y de América Latina, mediante el intercambio cruzado de conocimientos y experiencias.

\section{Crowdsourcing en BiblogTecarios: herramientas de comunicación interna y coordinación de la plataforma}

Para llevar a cabo el trabajo, BiblogTecarios recurre a tres aplicaciones de Google:

\section{a. Google groups}

Se ha creado un grupo en Google que sirve como vehículo principal de comunicación entre todos los miembros. Cualquier aspecto a tratar de forma colaborativa comienza mediante un mensaje colectivo: es el primer paso hacia la elaboración de entradas bajo el usuario colectivo "biblogtecarios" y posibles mejoras o nuevas implementaciones en el portal.

\section{b. Google calendar}

Permite la programación de todas las entradas en la plataforma. Se ha creado un calendario compartido, de manera que cada miembro reserva un día de publicación. Además, se añaden unas breves líneas sobre cuál será el tema de esa próxima entrada: esto permite evitar coincidencias en los temas a tratar en los posts.

El usuario Firmas invitadas permite publicar contenidos a los colegas que tienen algo puntual que contar o compartir

\section{c. Google docs}

La posibilidad de crear y modificar un documento simultáneamente, ver inmediatamente las anotaciones introducidas por otros, o abrir un chat sólo para discutir el documento compartido, hacen esta aplicación imprescindible para preparar entradas colectivas (como este mismo artículo).

Aun así, coordinar un grupo de personas siempre es complicado; más aún si se trata de un grupo heterogéneo, cuyos elementos no se conocían previamente entre sí, formado y funcionando en la nube y sin ninguna experiencia previa como equipo de trabajo.

A pesar de esta aparente dificultad, BiblogTecarios es un grupo coordinado. Ahora que ha alcanzado dimensiones más que considerables se ha hecho necesario crear grupos de coordinación encargados de gestionar aspectos concretos del trabajo grupal:

- Publicaciones: se ocupa de todo lo relacionado con las publicaciones que BiblogTecarios realiza, tanto en la propia plataforma bajo el usuario colectivo "BiblogTecarios", 
como en el exterior de la misma (congresos, jornadas, revistas...) Coordinadores: David Gómez-Domínguez y Evelio Martínez-Cañadas.

- Comunicación: se encarga de la comunicación corporativa y del correo electrónico colectivo. Interactúa con las distintas listas o grupos de distribución (por ejemplo IweTel) enviando los comunicados que se quieren hacer llegar al exterior. Coordinador: Diego Ariel-Vega.

- Relaciones internacionales: coordinadas por Diego ArielVega.

- Redes sociales: gestiona la imagen y la difusión de contenidos de BiblogTecarios y promueve debates en las redes sociales. Coordinadores: Belén Benito-Blázquez, Ana Doñate-Cifuentes, y Julián Marquina-Arenas.

A pesar de la constitución de estos grupos, cada uno de los miembros de BiblogTecarios sigue teniendo el compromiso de potenciar el grupo y hacer difusión mediante sus perfiles sociales personales.

\section{Redes sociales}

Desde BiblogTecarios se utilizan las siguientes redes sociales para completar la difusión de la plataforma:

\section{Twitter}

El perfil fue creado el 20 de julio de 2010, pero su primer tweet no se publicó hasta el 17 de septiembre, fecha en que se empezó a anunciar en qué consistía el nuevo proyecto. Twitter es una de las redes sociales más utilizadas y, gracias a las menciones y retweets, la plataforma está teniendo una mayor difusión. A día de hoy el perfil refleja unas interesantes cifras que día a día van en aumento (figura 2).

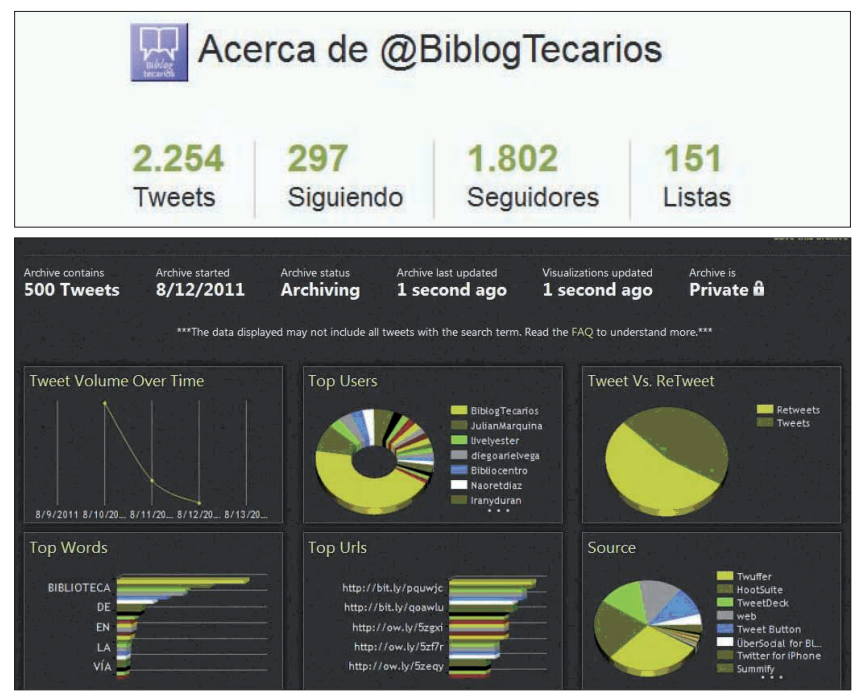

Figura 2. Estadísticas de las redes sociales de BiblogTecarios en Twitter

Dos herramientas complementan al perfil en Twitter: en primer lugar, TweetBook, una recopilación de tweets que se publica mensualmente; en segundo lugar, un canal de imágenes en Twitpic, de reciente creación, con el que se pretende compartir con los lectores imágenes que puedan ser de interés (como por ejemplo, la presentación sobre el Drupal de BiblogTecarios en Fesabid 2011).

\section{Facebook}

Página de fans creada próxima a la fecha de inauguración del portal, en octubre de 2010. Desde la página y por medio de la aplicación RSS Graffiti los post del portal se publican automáticamente.

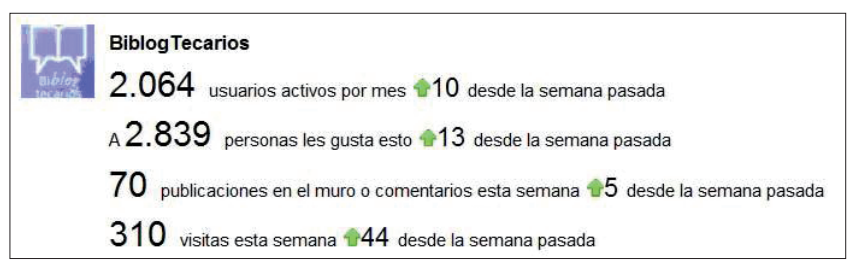

Figura 3. Estadísticas de las redes sociales de BiblogTecarios en Facebook

\section{YouTube}

Canal de vídeos, en el que se enlazan o marcan como favoritos aquellos que se consideran más interesantes y/o divertidos sobre el campo de la biblioteconomía y la documentación. El canal fue creado en octubre de 2010.

\begin{tabular}{|c|c|}
\hline Perfil & editar \\
\hline Nombre: & BiblogTecarios \\
\hline Reproducciones del canal: & 1683 \\
\hline \multicolumn{2}{|c|}{ Reproducciones totales de vídeos subidos: 24} \\
\hline Se unió el: & $30 / 09 / 2010$ \\
\hline Actividad más reciente & hace 4 semanas \\
\hline Suscriptores: & 25 \\
\hline Sitio web: & biblogTecarios.es \\
\hline \multicolumn{2}{|l|}{ Sobre mi: } \\
\hline \multicolumn{2}{|c|}{$\begin{array}{l}\text { BibliogTecarios es un espacio web colaborativo que } \\
\text { busca compartir noticias y opiniones sobre temas } \\
\text { de interés para los profesionales del mundo de la } \\
\text { Información y la Documentación. }\end{array}$} \\
\hline País: & España \\
\hline
\end{tabular}

Figura 4. Estadísticas de las redes sociales de BiblogTecarios en YouTube

Con el crecimiento de la plataforma se ha hecho necesario crear equipos de coordinación encargados de gestionar aspectos concretos del trabajo del grupo

Mencionaremos, por último, un producto relacionado con Twitter y Facebook: Diario de... BiblogTecarios. Fue creado mediante Paper.li, una aplicación web que recopila y estructura los enlaces compartidos en estas redes a modo de periódico. Tiene una periodicidad diaria, actualizándose a una hora determinada y su objetivo es mantener informado al 
lector de todo lo que ocurre en ambas redes sociales, de forma sencilla y organizada.

Las estadísticas de las redes de BiblogTecarios son más que satisfactorias, no sólo porque se han superado ampliamente las expectativas iniciales, sino también por la visibilidad que ha proporcionado a la plataforma (figuras 2, 3 y 4).

\section{Conclusiones}

El trabajo colaborativo en entornos en red cuenta con tres ventajas que confieren un valor añadido a este tipo de proyectos:

- se crean plataformas de opinión y debate dinámicos en los que se opina, se expone, se lee y se aprende de los autores y de los lectores que participan con la publicación de sus opiniones;

- estas plataformas constituyen una vía ideal de divulgación en el ámbito bibliotecario;

- se facilita la creación de redes personales y profesionales capaces de traspasar fronteras.

Gracias a las menciones y retweets en Twitter, la plataforma está teniendo una mayor difusión

BiblogTecarios es un ejemplo de cooperación y colaboración, que busca dar cabida a todos los aspectos y ramas posibles de la biblioteconomía y documentación. El hecho de que un conjunto de personas se reúnan virtualmente para aportar sus conocimientos y plasmarlos en un blog, es el resultado de las ganas de difundir información, y de la actitud emprendedora por parte de sus miembros.

\section{Miembros de BiblogTecarios}

- España:

Esther Angulo-Parejo (Barcelona); Ana Baeza (Albacete); María Benítez-Acosta (A Coruña); Belén Benito-Blázquez (Madrid); Irene Blanco-Faura (Madrid); Elena BoyadoAlonso (Madrid); Antonio Casado-Poyales (Toledo); María-José De-Acuña (Madrid); Ana Doñate-Cifuentes (Valencia); Natalia Garea (A Coruña); Manuela Gil-Bernabé (Alicante); David Gómez-Domínguez (Granada); Esti Gómez (Madrid); Noemí Gómez-Pereda (Cantabria); Jonathan González-Fernández (Asturias); Rebeca Hernández (Madrid); David Huélamo-Mercader (Madrid); Rafael Ibáñez-Hernández (Burgos); María Juanas (Madrid); Rubén Lamas-Recio (Vizcaya); David López-Higueras (Madrid); Antonio Maestre (Madrid); Julián Marquina-Arenas (Madrid); Amanda Marín-Mayol (Barcelona); Evelio Martínez-Cañadas (Barcelona); Fuensanta Martínez (Murcia); Manuela Martínez (Madrid); Laura Martínez-Alcaraz (Alicante); Sofía Möller (Barcelona); Juan-José Prieto (Madrid); Patricia Russo-Gallo (Barcelona); María-del-Carmen Sánchez-Arnedo (Madrid); Elena Sauri (Valencia); Cristian Serrano-Ramírez (Alicante); Roberto Soto-Arranz (León); Pilar Tornos (Valencia); Víctor Villapalos (Madrid).

- Argentina:

Jessica Castaño

Diego Ariel-Vega

- México:

Saúl Martínez-Equihua

- Colombia:

Jaider Ochoa

- Venezuela:

Estela Mastromatteo

- Puerto Rico:

Myma Lee

\section{Próximos temas centrales}

Enero 2012

Marzo 2012

Mayo 2012

Julio 2012

Septiembre 2012

Noviembre 2012
El futuro de la Web

Publicaciones científicas y acceso abierto

Organización del conocimiento

Comunicación digital

Información y derecho

Bibliotecas académicas

Los interesados pueden remitir notas, artículos, propuestas, publicidad, comentarios, etc., sobre estos temas a: http://recyt.fecyt.es/index.php/EPI/index 